\title{
Desimbricando la ciudad: crimen, inseguridad y organización espacial en Managua, Nicaragua
}

\section{Dennis Rodgers ${ }^{1}$}

1. Antropólogo de desarrollo urbano en el Departamento de Geografía y Medio Ambiente de la Escuela Londinense de Economía y Ciencia Política. Houghton Street, London WC2A 2AE, Gran Bretaña. e-mail: d.w.rodgers@lse.ac.uk

Recibido: septiembre 2005 / Aceptado: diciembre 2005

ESTE ARTÍCULO EXPLORA EL SURGIMIENTO DE UN NUEVO PATRÓN DE SEGREGACIÓN espacial vinculado al surgimiento de la inseguridad urbana en Managua, la capital de Nicaragua, durantela pasada década. Envés dela fragmentación en un archipiélago aislado de enclaves fortificados, como ha sido el caso de otras ciudades en todo el mundo, Managua ha sido sometida a un proceso mediante el que una porción completa de la metrópoli ha sido desmembrada de la estructura de la ciudad mediante la conformación de una exclusiva y fortificada red al servicio de las elites urbanas. Esta desmembración se fundamenta en la privatización de la seguridad y en la construcción de autopistas de alta velocidad e intersecciones. Este patrón urbano diverge significativamente de la Managua histórica y responde al nuevo desarrollo urbano, favorecido por el surgimiento directo e indirecto de las elites urbanas. En consecuencia, se plantea una pregunta crítica acerca de la naturaleza de las relaciones entre los grupos sociales ubicados en la ciudad.

Palabras clave: desarrollo urbano / urbanismo-Managua, Nicaragua / desarrollo de la comunidad / grupos sociales / interacción social

\section{Introducción}

La organización diferenciada del espacio es, desde hace mucho tiempo, un rasgo fundamental de la teoría urbana. El ejemplo tal vez más paradigmático es el modelo del crecimiento urbano por zonas concéntricas (Park, Burgess, McKenzie y Wirth, 1925), aunque la importancia de la organización espacial también es evidente en otras ideas sobre el desarrollo de las ciudades, incluyendo el surgimiento de los asentamientos marginales (Mangin, 1970), o los procesos de suburbanización (Jackson, 1985). El espacio es también un asunto clave en un creciente cuerpo de investigación relacionado con el surgimiento de lo que se ha denominado la "nueva segregación urbana" (Caldeira, 1999). Varios estudios desarrollados en diferentes sociedades de todo el mundo han manifestado patrones cambiantes de organización espacial urbana, ligados a los niveles de criminalidad e inseguridad crecientes ${ }^{2}$. El temor al crimen ha desembocado en el desarrollo de una nueva forma de organización espacial segregada en las ciudades, que 
se manifiesta, sobre todo, en la proliferación de los denominados “enclaves fortificados".

Según la definición de Teresa Caldeira (1999: 114), los enclaves fortificados son "espacios de residencia, consumo, recreo y trabajo que son privatizados, cerrados y monitoreados" . Son diseñados así para aislar a sus ocupantes del crimen y minimizar su inseguridad y, típicamente, toman la forma de condominios o barrios cerrados, con muros altos, tecnología sofisticada de vigilancia y seguridad privada de 24 horas, que protegen a los residentes y a las instalaciones. Al mismo tiempo, los enclaves fortificados pueden variar considerablemente. Por ejemplo, Maristella Svampa (2001) describe cómo en Buenos Aires, los llamados "los countries" pueden cubrir extensiones muy grandes, incluyendo hasta canchas de polo y de fútbol. Por contraste, en Santiago de Chile se constituyen irregularmente como "comunidades cerradas", por medio de la privatización de calles y plazas (Fischer, Jäger y Parnreiter, 2003). En ambos casos, son los opulentos quienes se aíslan. Esta es, obviamente, la situación más frecuente, pero todos los grupos sociales construyen muros, aunque también cuenta Jo Beall (2002) que en Johannesburgo (Sudáfrica), los trabajadores emigrantes, que viven en la pobreza, convierten sus albergues en el asentamiento de Soweto en "comunidades amuralladas”, para protegerse de la hostilidad de la sociedad.

A pesar de la variedad de sus formas, es generalmente reconocido que el surgimiento de enclaves fortificados transforma las ciudades, que dejan de ser espacios abiertos de libre circulación, para convertirse en localidades más fracturadas y fragmentadas, tipo archipiélago, lo que cambia de manera fundamental el carácter de la vida social urbana. Este nuevo patrón de organización espacial erosiona la propia noción de "espacio público", cómo explica Teresa Caldeira (1999: 130): "en una ciudad de muros y enclaves... el espacio público experimenta una profunda transformación. Sentido como más peligroso, fracturado por nuevos vacíos y enclaves, quebrado en sus viejas alineaciones... el espacio público... se abandona cada vez más a los que no tienen la oportunidad de vivir, trabajar e ir de compras en los nuevos enclaves privados, interiorizados y fortificados. Como... espacios..., están encerrados y vueltos hacia adentro, el espacio de afuera se deja para los que no tienen el dinero para entrar" . Los de "adentro" sienten poca responsabilidad hacia los de "afuera", porque no se relacionan con nociones de cohabitación e interacción, sino con un ideal de separación de los que perciben como distintos.

Esta lógica de separación espacial se interrelaciona frecuentemente con una lógica de exclusión social. En su estudio ya clásico del desarrollo urbano en São Paulo (Brasil), Teresa Caldeira (2000) observa cómo el retiro del espacio público hacia enclaves por parte de las clases altas de la ciudad, ha desembocado en el surgimiento de un discurso que asocia la criminalidad y la pobreza. Este discurso genera imágenes estereotípicas de los pobres como el "otro" que es intrínsicamente peligroso y sirve para legitimar su exclusión espacial de la vida de los ricos en el nombre de "seguridad", y engendra activamente formas de discriminación social. Los pobres están estigmatizados como "animales" imprevisibles y brutales que no merecen derechos humanos (ver Caldeira, 1996). Hay cada vez más llamadas para la reducción de los derechos civiles de los pobres y el patrullaje policial se enfoca cada vez más en las áreas pobres de São Paulo. Lo que David Harvey (2003) ha denominado el "derecho a la ciudad" se condiciona por atributos como la riqueza, la clase social o la residencia en determinada área. 
El presente articulo se ubica dentro de esta tradición de investigaciones urbanas y propone un análisis del desarrollo de una nueva forma de segregación social que se da en Managua, basado sobre datos recogidos por investigaciones desarrolladas en 1996-1997 y 20025. Aunque la ciudad presenta muchos de los clásicos rasgos del modelo de enclaves fortificados, el proceso de reorganización urbana parece presentar nuevos elementos, sobre todo cuando se considera desde la perspectiva de los pobres. En vez de fragmentarse en un archipiélago de islas de riqueza auto sostenientes y aisladas en un mar de pobreza, el espacio urbano de Managua ha experimentado un proceso en que todo un estrato de la metrópolis ha sido desconectado de la estructura general de la ciudad, de manera que quizás se puede hablar de un proceso de "desimbricación" urbana.

\section{Managua, la ciudad "palimpsesto"}

Quienes visitan Managua, frecuentemente oyen la expresión "Managua es Nicaragua", afirmación que tiene algo de verdad. En Managua vive casi la cuarta parte de la población nacional de 5.5 millones de personas, y más del $40 \%$ de la población urbana ${ }^{6}$. Domina el país en los aspectos económico y político y es el principal referente nacional en términos simbólicos. Sin embargo, Managua es también, en muchos aspectos, un elemento excepcional en el paisaje social y físico del país, siendo el punto focal de varios procesos y eventos únicos que la hicieron, y siguen haciéndola, un escenario muy particular.

En 1851, Managua, en aquel entonces un pueblo algo provincial y aletargado, fue seleccionada capital de Nicaragua, como compromiso entre dos ciudades más importantes, pero rivales y contendientes: León y Granada. En menos de un siglo, se había convertido en una metrópolis próspera de millón y medio de habitantes (no los había en el país en 1951) que durante los años sesenta tenía la reputación de ser un lugar de diversión para los ricos. Sin embargo, en 1972 la ciudad fue devastada por un terremoto que mató a 20,000 personas, destruyó el $75 \%$ de las viviendas y el 90\% de la capacidad comercial de la ciudad, incluyendo su animado centro, y dejó unas 300,000 personas sin hogar (Black, 1981: 57). Aunque mucha asistencia internacional afluyó a Nicaragua para contribuir con la reconstrucción, la mayor parte acabó en los bolsillos de la dictadura Somocista. Por eso, "la parte central destruida no fue reconstruida y... fue virtualmente abandonada. Sólo algunos edificios sobrevivieron al terremoto, y el centro adquirió un aspecto pos-apocalíptico... El esfuerzo de reconstrucción, que sí tuvo lugar después del terremoto de 1972, creó nuevas áreas residenciales al estesur-este del centro urbano... Esto presta a la ciudad la apariencia de un pulpo deforme. Los tentáculos del pulpo se extienden por grandes arterias de transporte desde el viejo centro, pero el cuerpo del pulpo es un conjunto de grandes hoyos" (Wall, 1996: 48-49). ${ }^{7}$

Aunque el derrocamiento de la dictadura Somocista en 1979 por la Revolución Sandinista resultó en planes ambiciosos para la transformación de Managua, la guerra civil con la Contra, que empezó inmediatamente después, agotó los recursos del nuevo Estado revolucionario, que preveía una reconstrucción a gran escala. Aunque se desarrollaron algunos proyectos para mejorar unos cuantos barrios pobres y asentamientos (ver Drewe, 1986; MINVAH, 1980; y Vance, 1985), esta actividad tuvo muy poco impacto en términos generales y la estructura de la ciudad se agravó aún más con el tiempo, a consecuencia del lento deterioro de la infraestructura urbana y el desarrollo anárquico de numerosos 
asentamientos, algunos de ellos ubicados en las ruinas del antiguo centro de la ciudad. Como describe Richard Leonardi (2001: 57) en una guía reciente de Nicaragua, Managua es una ciudad "sin centro, sin perfil y sin lógica".

Quizás no sea una sorpresa constatar que se refiere frecuentemente a Managua como "la ciudad del caos". Una alternativa sería considerarla como una metrópolis posmoderna, "un 'palimpsesto' de formas antiguas sobre impuestas unas sobre otras, y un 'collage' de usos actuales" (Harvey, 1990: 66) ${ }^{9}$. Los asentamientos de las ruinas del antiguo centro ejemplifican esta idea, pero la noción también puede ser aplicada de forma más general. Por ejemplo, los negocios y servicios que antes estaban en el centro han vuelto a surgir de manera descentralizada, creando una metrópolis fragmentada de distritos semi-autónomos, conectados por una red de transporte muy compleja. El ejemplo tal vez más ejemplificador es la manera como la población se adaptó al perfil pos-terremoto de la ciudad, trazando viejos puntos de referencia urbanos sobre la nueva ciudad. De este modo, frecuentemente se designan direcciones mediante referencias destruidas en 1972.

También se han dado nuevos cambios urbanos desde la derrota electoral de los Sandinistas en 1990. El regreso de los nicaragüenses opulentos que salieron hacia Miami después de la Revolución, desembocó en "esfuerzos decididos por parte de los 'Miami boy' (como son llamados)... para recrear su querido 'escenario' social y cultural de Miami” (Whisnant, 1995: 447) ${ }^{10}$ : bares, restaurantes y discotecas modernas hicieron su aparición, mientras por las calles de la ciudad empezaron a circular carros de lujo. Los procesos de globalización y de liberalización económica más amplios contribuyeron también a transformar Managua, especialmente, la llegada de franquicias internacionales como Subway, Pizza Hut, Hard Rock Café o McDonald's, junto con la construcción de varios hoteles considerados lujosos en el medio, supermercados exclusivos y la renovación al estilo estadounidense de dos centros comerciales de la ciudad, Plaza Inter y Metrocentro: ambos cuentan con cines con múltiples pantallas, áreas de comida rápida y tiendas donde se venden a precios inasequibles para el ciudadano medio, bienes de consumo importados: ropa de Benetton y Liz Claiborne, electrodomésticos de marca Sony, CDs y DVDs originales y navajas "Swiss Army" de Victorinox.

La gran mayoría de quienes se benefician con estos nuevos rasgos de la ciudad son los miembros de lo que se puede denominar, de forma general, las "elites urbanas"1. Aunque las recientes transformaciones de la ciudad pueden ubicarse dentro de la experiencia histórica de Managua como ciudad "palimpsesto", se puede argumentar que han sido complementadas y sostenidas por un proceso de cambio urbano cualitativamente distinto. Por contraste con el modelo de desarrollo urbano desorganizado del pasado, se puede señalar un proceso más ponderado de intervención a favor de las elites urbanas, que no ha buscado simplemente sobre imponer una nueva forma urbana sobre las del pasado, sino reestructurar activamente el perfil global de la ciudad mediante la separación explícita de ciertos espacios urbanos. Impulsando este nuevo modelo de segregación urbana, se encuentra el temor al crimen y a la inseguridad. 


\section{El crimen y la inseguridad en la Nicaragua de hoy}

El crimen se ha disparado en Nicaragua durante los últimos quince años, sobre todo en las zonas urbanas. Según datos de la Policía Nacional de Nicaragua, los niveles de criminalidad han aumentado constantemente a un promedio anual de 10\% desde el año 1990, muy superior al aumento anual de 2\% durante los ochenta (Serbin y Ferreyra, 2000: 185). El número absoluto de delitos más que se triplicó entre 1990 y 2003. Los delitos contra las personas, incluyendo homicidios, violaciones y asaltos, aumentaron en más de 460\% (véase Cuadro 1). Más cualitativamente, una encuesta realizada por CID-Gallup y divulgada en la edición correspondiente al 2 de mayo de 1997 del desaparecido periódico nicaragüense La Tribuna, encontró que una de cada seis personas entrevistadas manifestó haber sido víctima de un asalto criminal, por lo menos una vez en los últimos cuatro meses, proporción que aumentó a una de cada cuatro en Managua, donde se comete el 40\% de los delitos (Granera Sacasa y Cuarezma Terán, 1997: 32). En estas condiciones, no sorprende que, en otra encuesta realizada en 1999 por la ONG nicaragüense Ética y Transparencia, los encuestados mencionaron la criminalidad como el principal problema que enfrenta el país, por un margen de más del 30\% (PNUD, 2000: 130).

Cuadro 1. Estadísticas de delitos en Nicaragua

\begin{tabular}{|l|c|c|c|c|c|c|c|c|c|c|c|c|c|c}
\hline & $\mathbf{1 9 9 0}$ & $\mathbf{1 9 9 1}$ & $\mathbf{1 9 9 2}$ & $\mathbf{1 9 9 3}$ & $\mathbf{1 9 9 4}$ & $\mathbf{1 9 9 5}$ & $\mathbf{1 9 9 6}$ & $\mathbf{1 9 9 7}$ & $\mathbf{1 9 9 8}$ & $\mathbf{1 9 9 9}$ & $\mathbf{2 0 0 0}$ & $\mathbf{2 0 0 1}$ & $\mathbf{2 0 0 2}$ & $\mathbf{2 0 0 3}$ \\
\hline & & & & & & & & & & & & & & \\
\hline Delitos en general & 28005 & 30896 & 35924 & 42394 & 47173 & 48737 & 54983 & 62682 & 66040 & 72908 & 75741 & 90145 & 93497 & 97500 \\
\hline Incremento anual (total) & 1828 & 2891 & 5028 & 6470 & 4779 & 1564 & 6246 & 7699 & 3358 & 6868 & 2833 & 14404 & 3352 & 4003 \\
\hline Incremento anual (\%) & 7 & 10 & 16 & 18 & 11 & 3 & 13 & 14 & 5 & 10 & 4 & 19 & 4 & 4 \\
\hline & & & & & & & & & & & & & & \\
\hline $\begin{array}{l}\text { Delitos contra las } \\
\text { personas }\end{array}$ & 7340 & 9392 & 12072 & 13089 & 15500 & 17934 & 19821 & 23824 & 25804 & 29824 & 26546 & 32011 & 33519 & 33961 \\
\hline Incremento anual (total) & 996 & 2052 & 2680 & 1017 & 2411 & 2434 & 1887 & 4003 & 1980 & 4020 & -3278 & 5465 & 1508 & 442 \\
\hline Incremento anual (\%) & 16 & 28 & 29 & 8 & 18 & 16 & 11 & 20 & 8 & 16 & -11 & 21 & 5 & 1 \\
\hline & & & & & & & & & & & & & & \\
\hline Homicidios (total) & 672 & 732 & 828 & 762 & 725 & 707 & 662 & 679 & 639 & 561 & 476 & 537 & 591 & 664 \\
\hline Incremento anual (total) & 159 & 60 & 96 & -66 & -37 & -18 & -45 & 17 & -40 & -78 & -85 & 61 & 54 & 73 \\
\hline Incremento anual (\%) & 31 & 9 & 13 & -8 & -5 & -2 & -6 & 3 & -6 & -12 & -15 & 13 & 10 & 12 \\
\hline & & & & & & & & & & & & & & \\
\hline $\begin{array}{l}\text { Tasa de homicidio } \\
\text { por 100,000 habitantes) }\end{array}$ & 18 & 19 & 20 & 18 & 17 & 16 & 15 & 15 & 13 & 11 & 9 & 10 & 11 & 12 \\
\hline
\end{tabular}

Fuentes: Granera Sacasa y Cuarezma Terán (1997: 37-49) y Serbin y Ferreyra (2000: 185-7) para los datos de 1990-1992. Las estadísticas de 1993-2003 son del sitio Internet de la Policía Nacional de Nicaragua, consultado el 18 de junio de 2004 (http:/www.policia.gob.ni/estadisticas/ dosier2003/dosier4.htm, http:/www.policia.gob.ni/estadisticas/dosier2003/dosier5.htm y http:// www.policia.gob.ni/estadisticas/2002/anuario/indicadores/adnivelnacional_tipologia_anos.htm). La tasa de homicidio fue calculada utilizando datos demográficos del PNUD (2000 y 2002) y del sitio internet del Instituto Nacional de Estadísticas y Censos, consultado el 18 de junio de 2004 (http://www.inec.gob.ni/estadisticas/proyeccion2002.htm y http://www.inec.gob.ni/estadisticas/ proyeccion2003.htm). 
Aunque la tendencia global ascendente de la criminalidad refleja seguramente la realidad, las estadísticas oficiales de la Policía Nacional son problemáticas. Como señalan Godnick, Muggah y Waszink (2002: 26), "dada la información anecdótica sobre la violencia tal como está representada en la prensa y la percepción general de la violencia dentro de la sociedad nicaragüense, estos datos son sospechosamente bajos"12. La tasa nacional de homicidios por cada 100 mil habitantes, el parámetro aceptado internacionalmente para medir niveles de violencia, es particularmente problemático en el contexto regional. El promedio entre 1990 y 2003 era de 15 muertos por 100 mil personas, frente a una tasa casi tres veces más alta en Honduras y más de seis veces más alta en Guatemala y El Salvador, según Moser y Winton (2002: 47). Aunque Nicaragua es, sin duda, menos violenta que Honduras, Guatemala o El Salvador, es muy violenta, especialmente en sus áreas urbanas. Durante una estancia en Managua entre 1996 y 1997, tuve conocimiento personal de nueve homicidios en determinado barrio pobre, lo que equivale a 360 muertos por 100 mil personas. Aunque hay que tomar esta cifra con mucho cuidado, porque no es sistemática ni está basada en una muestra significativa, por lo menos indica que hay que manejar las estadísticas oficiales con cierto escepticismo (ver Rodgers, 2006).

Hay varias explicaciones para la naturaleza problemática de las cifras oficiales. La primera consiste en que el presidente anterior, Arnoldo Alemán (1997-2001) y el actual, Enrique Bolaños convirtieron la lucha contra el crimen en un elemento fundamental de sus programas de gobierno y les es más conveniente tener estadísticas positivas. Es decir, reducidas. Pero tal vez la razón más importante es la ineficiencia y debilidad de las instituciones estatales nicaragüenses. La Organización Panamericana de Salud (1998: 384) calcula que más del $50 \%$ de las defunciones ocurridas en Nicaragua durante el año 1995 no se registraron, a causa del registro deficiente por parte de los hospitales y la falta de conocimiento, entre la población, acerca de dónde registrar acontecimientos como la muerte de familiares.

Pero la debilidad institucional más grave se refiere, posiblemente, a la Policía Nacional. Desde el cambio de régimen en 1990, su capacidad institucional y su eficacia se han visto severamente afectadas por un lento proceso de despartidarización y de reducción de su tamaño y presupuesto, en parte relacionado con los esfuerzos del estado nicaragüense para cumplir con las rigurosas condiciones del ajuste estructural impuestas por el Fondo Monetario Internacional (FMI) y el Banco Mundial (BM).

Según un estudio de Roberto Cajina (2000: 174), la Policía Nacional tiene limitada capacidad de patrullaje en las zonas urbanas y está completamente ausente en el 21\% de los 146 Municipios del país. Además, la Policía tiene, regionalmente, el número más bajo de agentes per cápita y por delito, el presupuesto más bajo por delito y por agente, y los salarios promedios más bajos en América Central (Call, 2000: 24-5). Tal penuria, obviamente, deja a los agentes policiales muy susceptibles a la corrupción, y significa una clara limitación en cuanto a sus capacidades técnicas y materiales (Grigsby, 2003). En unas declaraciones a los medios en 2001, el comisionado Franco Montealegre admitió que, a menudo, la policía se encontraba con menos potencia de fuego que los delincuentes, sobre todo frente a las pandillas, que son muy fuertes en las áreas urbanas ${ }^{13}$. En estas circunstancias, no sorprende que el 43\% de los encuestados que dijeron ser víctimas de algún delito en la encuesta de 1999 de Ética y Transparencia, afirmaron no haber reportado el delito a la Policía, porque 
“no servía para nada” (Cajina, 2000: 178).

A pesar de la falta de fiabilidad de las estadísticas policiales oficiales, los altos niveles de criminalidad en la Nicaragua urbana eran indudables durante las investigaciones realizadas para este trabajo, a nivel de barrio, entre 1996-1997 y en 2002. De hecho, se manifestaba un evidente temor a salir del hogar en 1996 y la gente salía lo menos posible, restringiéndose a algunas rutas y destinos fijos. En 2002, hasta el refugio de la casa parecía precario, y la gente casi se atrincheraba en su casa. Una habitante de un barrio, llamada Adilia, describió la situación en 1997 como "casi vivir en un estado de sitio", y en 2002 dijo que "la situación está aún peor, la gente tiene miedo de salir de su casa, es demasiado peligroso". Su madre, doña Yolanda, le hizo coro de forma dramática: "Hoy en día hay tanta delincuencia que no se puede vivir. Por un reloj te matan. Por un zapato te matan. Por robarte un pantalón, te matan. Hoy tiene que desconfiar uno del propio vecino, del propio amigo que fue, o es también. Pero ese amigo te puede robar... Nunca sabes... ¿Por qué está pasando esto? Ya no podés salir a pasear, ya no podés andar un anillo, una pulsera, un buen zapato, algo que uno pueda presentar para vivir un poco como personas de la sociedad, algo que nos hace parecer un poco mejor que somos... ¿Como podemos vivir? Uno no puede..."

Para las elites urbanas, sin embargo, la situación evolucionó de forma muy distinta, como ilustran las dos anécdotas que siguen. La primera tiene por protagonista a un hombre evidentemente rico que volaba de Miami a Nicaragua en 1996. Cuando supo que el autor de este trabajo planificaba permanecer durante un año en Managua, manifestó su desacuerdo pues, según su criterio, la ciudad era demasiado peligrosa, con elevados niveles de criminalidad y violencia, con frecuentes asaltos en los semáforos, con calles mal asfaltadas donde era casi inevitable sufrir algún percance mecánico que podía resultar en un asalto, y donde no había lugares seguros y agradables para comer, beber, bailar o divertirse. Por último, manifestó que regresaba de Miami a donde había viajado en búsqueda de una casa para mudarse con su familia lo antes posible. La segunda anécdota también tuvo por escenario un avión que volaba de Miami a Managua, pero sucedió cinco años más tarde, en 2002, en viaje para complementar la primera investigación. También el protagonista de la historia es un nicaragüense opulento quien, en extraña simetría con la anécdota anterior, tras ocho años de residir en Miami, estaba mudándose a Managua pues, según su criterio, la ciudad se había transformado, gracias al presidente Arnoldo Alemán, en un lugar seguro y habitable, con buenos restaurantes, bares, hoteles y casinos.

\section{Nueva Managua}

Evidentemente, Managua ha cambiado mucho, pero los cambios son muy diferentes dependiendo de la perspectiva. Según doña Yolanda, entre 1997 y 2002 “para los pobres, nada ha cambiado salvo que ya han pasado cinco años y no se mejoró el futuro...”. Es obvio que las formas predominantes de violencia urbana afectan a los pobres y las elites urbanas de forma muy desigual. A diferencia de la percepción del protagonista de la segunda anécdota, el crimen y la inseguridad parecen haber empeorado en los barrios pobres. Según doña Isabel, otra habitante del barrio, "para los ricos es distinto, la vida se ha vuelto más fácil, ahora la ciudad es como su ciudad, hasta que uno podría decir que para los ricos es como si hubiera una nueva Managua”. 
Varios factores explican la transformación desigual de Managua, pero no hay duda que Arnoldo Alemán jugó un papel importante: fue elegido Alcalde de la ciudad en 1990 y llegó al poder con un proyecto urbano muy bien definido para Managua, enfocado en transformar la ciudad en un lugar más cómodo para las elites urbanas. Esto se tradujo en una serie de obras públicas ostentosas para "embellecer" la ciudad, incluyendo una rotonda de gran tamaño, con una fuente grande que, cuando está iluminada, parece arrojar chorros de agua de distintos colores; la reconstrucción del malecón de Managua; y la construcción de una nueva y enorme catedral. Otras iniciativas incluyeron campañas regulares para sacar de los semáforos a niños de la calle y vendedores, borrar los murales realizados por artistas revolucionarios y arrasar varios asentamientos en las ruinas del centro de la cuidad.

Cuando Alemán fue elegido Presidente en 1996, continuó su campaña para transformar Managua, pero con más recursos. Supervisó personalmente la construcción de las nuevas oficinas gubernamentales en el antiguo centro de la ciudad, incluyendo, por un costo de cuatro millones de dólares donados por Taiwán (Díaz Lacayo, 1998), un nuevo Palacio Presidencial con una fuente cuyos chorros de agua se mueven al ritmo de melodías computerizadas. El viejo aeropuerto internacional de Managua fue también completamente renovado entre 2000-2001, por un costo total de 33.4 millones de dólares (INIFOM, 2003: 29). El gobierno nacional estimuló nuevas construcciones de manera indirecta al ofrecer exenciones fiscales, - ilegales -, a compañías dispuestas a levantar nuevos edificios. El Grupo Pellas gastó 20 millones de dólares en la construcción de una torre ultra-moderna de 14 pisos, y recibió una exención fiscal de 2.5 millones de dólares (Chamorro, 2002). Exenciones similares también se otorgaron a los grupos de Taiwán y El Salvador que construyeron los centros comerciales de Plaza Inter y Metrocentro, respectivamente.

Es obvio que los esfuerzos de "embellecimiento" realizados por Alemán se enfocaron principalmente en la creación de locales que facilitan de forma directa la vida de las elites urbanas, tales como las oficinas gubernamentales o el aeropuerto internacional ${ }^{14}$. Cuando se consideran estas manifestaciones de desarrollo junto con los nuevos y exclusivos bares, restaurantes y centros comerciales que surgieron durante los años noventa, se puede sostener que en Managua emergió todo un conglomerado de locales y servicios principalmente para las elites de la ciudad. Sin embargo, como se quejaba el protagonista de la primera anécdota en 1996, esto no era suficiente para hacer que la ciudad resultara atractiva a las elites urbanas a causa de los crecientes niveles de criminalidad e inseguridad. Lo que se requería era una reorganización mucho más enérgica del orden urbano, que parece haberse fundamentado, principalmente, en dos factores: el desarrollo de la seguridad privada y el mejoramiento de la infraestructura vial.

\section{La privatización de seguridad}

El desarrollo de la industria de la seguridad privada en la Nicaragua urbana ha coincidido con los crecientes niveles de criminalidad e inseguridad, y existe una opinión generalizada de que la ineficiencia de la Policía hace necesarias las soluciones privadas para garantizar la seguridad. En 1990, en la Policía sólo había registrada una empresa privada de seguridad; en 1996 ya había 14 que llegaron a 56 en 2003. En total, nueve mil 17 guardias de seguridad privados estaban registrados en 2003, aunque es probable que el número real sea mucho más 
alto, considerando que se concedieron 29 mil 414 licencias de armas de fuego a empresas de seguridad privada en 2000. En comparación, había un total de siete mil 664 policías en $2003^{15}$. Por supuesto, como con las estadísticas oficiales del delito, hay que tener cuidado con estas cifras y verlas más como indicadores de tendencias que como datos exactos de la realidad nicaragüense. La Policía Nacional admite que no tiene un registro completo de las empresas de seguridad privada en el país; además, acepta que su registro de armas de fuego es deficiente por lo menos en el 50\% (Godnick, Muggah y Waszink, 2002: 4).

Sin embargo, la seguridad privada es un rasgo clásico del modelo de segregación urbana de los enclaves fortificados. Como explica Caldeira (2000: 258-59), el retiro temeroso de las elites en São Paulo hacia barrios y condominios cerrados, se acompaña, inevitablemente, de una necesidad de asegurar la exclusión de criminales potenciales en estos espacios. En virtud de su naturaleza privada, los enclaves fortificados no pueden contar necesariamente con el apoyo de las organizaciones de seguridad públicas para imponer esta exclusión, y la seguridad se transforma entonces en un asunto privado. Florence Babb (2001: 67-68) sostiene que la misma lógica se aplica en Managua, dónde "los ricos... se protegen del crimen y otros problemas sociales, construyendo los muros más altos que pueden, comprando los mejores sistemas de seguridad para sus hogares y pagando a guardias armados para patrullar sus vecindades. De esta manera, crean enclaves segregados que, en Managua como en otros lugares de América Latina, cambian el carácter del espacio público y de la vida pública e imponen reglas de inclusión y exclusión... [en] las calles de Managua... dejadas a los que no tienen el dinero para retirarse a los enclaves" ${ }^{\prime 16}$.

Las observaciones de Babb tienen algo de verdad y algo de error. Es evidente que han proliferado muros altos y guardias armados en Managua durante la última década pero, al mismo tiempo, estas medidas de seguridad afectan más a las residencias individuales que a vecindades enteras. Los pocos sectores ricos que existen en Managua no son barrios cerrados como los descritos por Caldeira (2000), sino concentraciones de viviendas individualmente fortificadas. Se puede especular que el peso numérico relativamente reducido de las elites urbanas de Managua hace que los barrios cerrados no sean muy viables. De manera clásica, esas comunidades son espacios cuyos residentes casi no necesitan salir, ya que contienen todos los servicios sociales, económicos y culturales que requieren (Low, 2003). Eso significa que las empresas involucradas sirven mercados aislados que, necesariamente, tienen que tener un cierto tamaño para ser viables, porque son exclusivos. Pero las elites urbanas de Managua tienen un tamaño reducido, lo que significa que los enclaves tienen que tener un tamaño inevitablemente modesto. En consecuencia, la rentabilidad de las empresas que sirvan a esos enclaves puede ser problemática.

Sea o no esta restricción demográfica la causa de la ausencia de comunidades cercadas en Managua, lo cierto es que las elites urbanas no se han retirado del espacio público de la misma manera que lo han hecho los ricos en São Paulo. En vez de vivir en comunidades cerradas, salen de sus hogares individualmente fortificados para trabajar y divertirse en las nuevas oficinas gubernamentales, los nuevos edificios de negocios, y los nuevos bares, restaurantes y centros comerciales de Managua. A este nivel, la seguridad privada es un factor crítico, porque su presencia alrededor de estos locales, convierte estos espacios públicos en espacios privados de facto, como explica doña Yolanda: "todas esas tiendas y 
centros comerciales bonitos no son para los pobres, las guardias no te dejen entrar si no sós rico, todos te miran mal, porque sós pobre y no te quieren allá..."

Puesto que los espacios en los que viven las elites urbanas de Managua se encuentran dispersos por toda la metrópolis, es plausible describirlos como una "red fortificada". Al igual que los enclaves fortificados, una red fortificada está separada del resto de la ciudad y permite que las personas de adentro permanezcan aisladas de los altos niveles de criminalidad e inseguridad urbanos que caracterizan el resto de la ciudad. Al mismo tiempo, es la interconexión de estos espacios protegidos de forma privada lo que los hace un "sistema" viable, y se puede argumentar que el elemento más crítico en el surgimiento de esta "red fortificada" ha sido el desarrollo, durante los últimos cinco años, de un conjunto estratégico de calles bien-mantenidas y bien iluminadas que permiten el rápido desplazamiento a través de Managua.

\section{Calles y rotondas}

En un artículo reciente que llama a la elaboración de una verdadera "economía política de calles", Fiona Wilson (2004: 529) sostiene que "en vez de considerar las calles como líneas neutrales... que van desde... el punto $a$ al punto $b$, deberían ser visualizadas como lugares estirados donde se agrupan y se adhieren relaciones sociales confluyentes"17. En 1996, las calles de Managua, con sus baches y su falta de revestimiento adecuado, y con el riesgo constante de asaltos en los semáforos y encrucijadas, durante la primera mitad de los años noventa, convirtieron en un riesgo constante para las elites urbanas viajar entre las distintas locaciones de la ciudad. Estas elites, aunque podían proteger sus casas, oficinas y bares, no podían evitar encuentros potencialmente peligrosos cuando se desplazaban entre los diferentes puntos, espacialmente dispersos, donde se desarrollaban las diferentes facetas de sus vidas. En otras palabras, a pesar de la proliferación de lugares exclusivos en Managua, estos continuaban ubicados dentro de la ciudad y sus ocupantes y usuarios ricos estaban obligados a convivir con los demás habitantes de la metrópolis, lo quisieran o no.

En esas condiciones, no debe sorprender que Alemán haya dirigido sus esfuerzos a transformar la red de transporte de Managua. En 1998 se inició un programa de gran escala para rellenar baches, revestir y ampliar las carreteras más importantes de la metrópolis, construir una carretera de circunvalación en el suroeste de la ciudad, y reemplazar los semáforos con rotondas, para acelerar el movimiento del tráfico y reducir la congestión. Sin embargo, la proliferación de rotondas también se puede vincular a la reducción del riesgo de asaltos a los vehículos, pues no tienen que detenerse; mientras que el propósito primario de la carretera de circunvalación parece haber sido permitir a los chóferes evitar una parte de Managua particularmente reputada por sus altos niveles de delincuencia. En general, es un patrón que sugiere una construcción o reconstrucción bastante selectiva. Las obras de vialidad parecen tener el objetivo de conectar los lugares donde se desarrolla la vida de las elites urbanas, mientras las calles de las áreas de la ciudad que no están asociadas con las elites urbanas, como el mercado Oriental, han sido casi totalmente abandonadas ${ }^{18}$.

Esas mejoras selectivas de la ciudad continuaron aún después de que Managua cambiase de manos políticas en el 2000, con la elección a la Alcaldía del sandinista Herty Lewites. Aunque 
Lewites propuso un programa más equitativo de desarrollo urbano, que incluía seis millones de dólares en obras viales en 2001, el muy reducido presupuesto municipal hizo necesario el apoyo financiero del gobierno nacional, encabezado por Alemán, para su realización, y no le fue concedido ${ }^{19}$. Sin embargo, al mismo tiempo, el gobierno nacional financiaba importantes mejoras en los 45 kilómetros de la carretera a Masaya, por un costo de 25.8 millones de dólares ${ }^{20}$, lo que sin duda estaba muy relacionado con el creciente número de familias de las elites urbanas que han trasladado su residencia al área suburbana comprendida entre Managua y Granada, quienes necesitan entrar y salir de Managua de forma rápida y segura. La misma lógica se puede aplicar para explicar por qué se construyeron 13 rotondas mayores en Managua durante la última década, mientras que el plan de Lewites para aumentar la minúscula cantidad de semáforos de la ciudad de 78 a 259 (INIFOM, 2003: 26), ha recibido poco apoyo. En vez de eso, el Ministerio de Transporte e Infraestructura y la Policía Nacional propusieron una campaña para educar a los chóferes sobre el uso adecuado de rotondas...

Aunque se puede argumentar que muchos de los cambios recientes Managua relacionados con el transporte favorecen a las elites urbanas, conviene saber cómo los perciben los pobres para comprender su verdadero significado. Como señaló doña Yolanda en 2002, los pobres no sólo han sido excluidos de los hogares, oficinas y locales de ocio de las elites urbanas, sino también de los espacios de conexión entres estos lugares; es decir de las mismas calles y rotondas:

"Todo lo que ha hecho Alemán, lo ha hecho para los ricos. Todo es grande, de lujo, estilo americano. Si vos te vas a ver la rotonda de la Purísima allá arriba, es una rotonda grandísima ienorme! Vas a ver la de Jean-Paul Genie, es grandísima también. Vas a ver la del Guegüense, otra rotonda. Vas a ver la del Huembes, otra rotonda. También está mejorando la Carretera a Masaya, ahora tiene tres líneas de carros, ida y vuelta... Pero la cosa es que no estamos viviendo en los Estados Unidos, estamos viviendo en la Nicaragua pobre, en miseria, porque no hay sueldos... Solo al rico le gusta andar en esas rotondas, luciendo sus Cherokees, luciendo sus camionetas grandes. Han echado más de tres millones de carros en las calles, ¿́pero de quiénes son esos carros? Los pobres no tienen para comprar Cherokees y camionetas, ipor supuesto que no! Entonces, ¿qué nos han traído para nosotros esas nuevas carreteras y rotondas? iNi verga! Los buses que usan los pobres todavía pasan por las calles viejas, quebradas y llenas de hoyos... Las calles no son para los pobres, digo yo... Además, no es posible caminar por ningún lado con todos esos carros grandes que andan por las calles tan rápido. ¿Trataste de cruzar una de esas calles? Es imposible, sobre todo en las rotondas adonde no sabes de donde pueden salir los carros. Antes estaba más lento el tráfico, y no había tanto, pero ahora... ¿Vos conociste a doña Aurelia, la que vive como tres casas más abajo? Mataron a su hijo hace unos meses cuando estaba intentando cruzar la calle. El carro ni siquiera se detuvo, le golpeó y se fue sin parar... Fue como si nos estuvieron diciendo que las calles no son de nosotros, sino de ellos... Es como si hubieran arrancado los pedazos de la ciudad que quieren para ellos y que fuera prohibido que las usáramos nosotros, los pobres..."

Aunque no son tres millones de nuevos carros los que han aparecidos en las calles de Managua, es cierto que la cantidad de vehículos automotores ha aumentado de manera desmesurada en Nicaragua desde finales de los noventa. Hay más de 200,000 carros en 
Nicaragua y hubo un incremento de 35\% del parque automotor entre 1998 y 2001 . La gran mayoría de esos nuevos vehículos son privados, más del 70\% eran carros y camionetas entre 2001 y 2002 y más del 60\% de todos los vehículos se concentran en la ciudad capitalina (INIFOM, 2003: 24) ${ }^{21}$. No sorprende que, tal como lo sintió doña Yolanda, parece que hay más muertes por accidentes en las nuevas calles. Aunque las cifras de transporte de la Policía Nacional deben ser tratadas con la misma prudencia que sus estadísticas criminales, sí muestran un aumento repentino en las muertes por accidente de tráfico en Managua entre 1998-2000, lo que coincide con los más importantes cambios en la red de transporte de la cuidad. Ciertamente, de las tres calles con mayor número de accidentes de tráfico, dos son carreteras nuevas (INIFOM, 2003: 25). Además, los peatones representan el grupo más grande de víctimas de tráfico con un total del 40\%22. Las rotondas son lugares particularmente peligrosos para los peatones, aunque se debe también reconocer que hay apenas dos pasos de peatones en todo el conjunto de Managua, solamente algunos puentes peatonales y pocas carreteras $^{23}$. En general, todos estos nuevos peligros han implicado para los pobres, - la gran mayoría en Managua -, una creciente circunscripción de los espacios de la ciudad y más dificultades para desplazarse en el ámbito urbano.

\section{“Desimbricando” la ciudad}

Como afirma Alan Smart (2001: 30), "todas las ciudades intentan gobernar sus espacios constitutivos y a quienes viven en ella, aunque en distintos grados" ${ }^{24}$. La pregunta es cómo lo hacen y con qué propósito. En un artículo ya clásico de estudios urbanos, Sally Engle Merry (2001: 16) describe como la gobernabilidad de las ciudades se interesa cada vez más en el manejo del espacio, en vez de sancionar a los infractores de las reglas de convivencia metropolitana. En otros términos, hay una transición desde los modelos de gobernación disciplinarios hacia los modelos de gobernación espacial. Eso aplica tanto al nivel privado como al público y el ejemplo clásico de esta nueva forma de gobernabilidad espacial son los enclaves fortificados observados por Caldeira (2000) y otros en todo el mundo, que generan orden creando espacios de calma por medio de exclusión, y no mediante campañas activas de represión de los comportamientos que se consideran "ofensivos". Vistas así las cosas, hay muchos paralelismos entre el modelo de desarrollo urbano constituido por los enclaves fortificados y la transformación de Managua durante la última década y media. Pero al mismo tiempo, también se aprecia que la nueva Managua presenta varios elementos distintos e intrigantes.

Los enclaves fortificados son mundos desconectados, la antítesis del espacio público, desde el instante en que constituyen un espacio retirado de la estructura de la ciudad, que resulta fragmentada en islas. Sin embargo, el nuevo orden espacial de Managua ha sido establecido, no tanto a través del retiro insular de la ciudad, sino a través de la constitución de una red fortificada que se extiende por la faz de la metrópolis. Por supuesto, esa red fortificada constituye una forma de desconexión de la estructura general de la ciudad en su totalidad, en el sentido de que es un espacio exclusivo que existe sólo para las elites urbanas, pero su extensión geográfica hace que no se puede hablar de fragmentación en el mismo sentido que tiene los enclaves fortificados. Más que una fragmentación, es tal vez mejor describir el proceso experimentado por Managua como una forma de “desimbricación”. 
Este término es un préstamos del inglés, “disembedding”, de Anthony Giddens (1990; ver también 1991 y 1999), quien lo utiliza para describir cómo las relaciones sociales pueden separarse de sus contextos localizados por los efectos de la modernidad y de la globalización. Giddens está especialmente interesado en explorar el fenómeno en los niveles cultural y económico, pero también se puede decir que la noción de "desimbricación" tiene dimensiones territoriales, sobre todo en relación con "la medida en que un actor está 'anclado' en territorios o lugares particulares" (Hess, 2004: 177) ${ }^{25}$. Desde este punto de vista, las ciudades constituyen sitios primordiales de imbricación territorial que, al ser crisoles sociales, moldean de manera fundamental las formas por las cuales viven los actores sociales dentro de su ámbito.

La historia urbana de Managua como "palimpsesto" ejemplifica claramente este proceso, con nuevas formas urbanas que adoptan, reinterpretan y son formadas por las viejas. Sin embargo, podría decirse que la reciente transformación espacial de Managua ha desembocado en una forma social urbana que ya no se relaciona con su contexto territorial más amplio y a la que se puede aplicar la etiqueta de "desimbricada”. La red fortificada de las elites urbanas excluye a los otros, no sólo de lugares aislados específicos, sino también de las calles y las encrucijadas de la ciudad que conectan dichos lugares. Al hacerlo, se quiebra activamente el espacio público de la ciudad de forma mucho más extensiva que como lo hacen los enclaves fortificados, "arrancando", - para utilizar la expresión de doña Yolanda -, grandes áreas de la metrópolis para el uso exclusivo de las elites urbanas, tanto directamente como indirectamente, impidiendo el movimiento de la gente dentro de la ciudad. Como indica Caldeira (2000: 213), esta dinámica toma tal vez su expresión más significativa cuando se la considera en términos de "cómo se relacionan los grupos sociales unos con otros, en el espacio de la ciudad" (ver también Harvey, 1973) ${ }^{26}$. Esta perspectiva enfoca la atención directamente sobre preguntas acerca del gobierno urbano y, sobre todo, en la cuestión de para quién y por quién se realiza.

Durante la última década y media, el gobierno de Managua ha favorecido claramente a las elites urbanas. Los recursos se han utilizado para satisfacer las necesidades y deseos de los más ricos, sin tener en cuenta el resto de la ciudad. La ciudad ha sido remodelada a su imagen y semejanza, y giran en torno a sus prioridades. Vistas así las cosas y puesto que quienes se benefician de la transformación de la metrópolis son también quienes producen esos cambios, es posible suponer que la "desimbricación" de Managua representa un ejemplo de lo que Christopher Lasch (1995) denomina "la sublevación de las elites". Tras una década de gobierno popular revolucionario, seguida por una ola de crimen y delincuencia desenfrenados - ¿que correspondería a una "sublevación de las masas" à la José Ortega y Gasset (1985[1932])? -, las elites urbanas nicaragüenses han decidido tomar su propio rumbo, no tanto retirándose de la ciudad, sino más bien dividiéndola y estableciéndose de manera autónoma dentro de su propio espacio autodeterminado y “desimbricado". Al hacerlo, sin embargo, "traicionan" activamente el contrato social implícito en la ciudad, que deriva del hecho de estar incrustados en un espacio urbano común e, inevitablemente, producen "mundos de desigualdad, alienación e injusticia" (Harvey, 2003: 941)27. 


\section{Notas}

1 Antropólogo, profesor de Desarrollo Urbano en el Departamento de Geografía y Medio Ambiente de la Escuela Londinense de Economía y Ciencia Política. Sus intereses académicos incluyen la violencia en Nicaragua y la gobernabilidad urbana en Argentina. Ha realizado investigaciones sobre pandillas juveniles, pobreza, desarrollo urbano, movimientos sociales, presupuesto participativo y ha publicado varios artículos sobre estos temas, tanto en inglés como en español. Dirección: Department of Geography and Environment, London School of Economics, Houghton Street, London WC2A 2AE, Gran Bretaña. Teléfono: +44 (0)20 7955 7718, Fax: +44 (0)20 7955 7412. Correo electrónico: d.w.rodgers@|se.ac.uk. Este artículo, traducido por Gareth Richards, de la revista Envío (Managua, Nicaragua) y revisado por el autor, fue publicado originalmente en el volumen 16, número 2 de la revista Environment and Urbanization (Londres, Gran Bretaña) en octubre de 2004. El autor agradece a Claire La Hovary, Caroline Moser, José Luis Rocha y Peter Sollis por sus valiosos consejos y comentarios, así como también a Jo Beall y Mike Davis por su apoyo alentador.

2 Ver, por ejemplo, Beall (2002), Blakely y Snyder (1997), Borsdorf (2002), Caldeira (1996, 1999 y 2000), Connell (1999), Davis (1990 y 1998), Fischer, Jäger y Parnreiter (2003), Low $(2001,2003)$, Marcuse (1997a y 1997b), Sabatini y Arenas (2000), Salcedo y Torres (2004), y Svampa (2001).

3 Texto original: "privatized, enclosed, and monitored spaces of residence, consumption, leisure, and work".

4 Texto original: "in a city of walls and enclaves..., public space undergoes a deep transformation. Felt as more dangerous, fractured by the new voids and enclaves, broken in its old alignments, ...public space ... is increasingly abandoned to those who do not have a chance of living, working, and shopping in the new private, internalised, and fortified enclaves. As ....spaces ....are enclosed and turned inside, the outside space is left for those who cannot afford to go in."

5 La primera investigación fue desarrollada entre julio de 1996 y julio de 1997, para el Doctorado del autor en Antropología Social, obtenido en 2000 de la universidad de Cambridge, Inglaterra (ver Rodgers, 2000). La segunda investigación, desarrollada entre febrero y marzo de 2002 y diciembre de 2002, era parte de un proyecto más grande sobre criminalidad y violencia en América Latina, patrocinado por el Programa de los Estados de Crisis de la Escuela Londinense de Economía y Ciencia Política (ver http://www.crisisstates.com).

6 Ver los datos en línea del Instituto Nicaragüense de Estadísticas y Censos (INEC) por el sitio Internet http://www.inec.gob. ni/estadisticas/proyeccion2003.htm, consultado el 20 de febrero de 2004.

7 Texto original: "the destroyed central part of Managua was not rebuilt and ....was virtually abandoned. Only a few buildings survived the earthquake, and the central core took on a post-apocalyptic look. ...The rebuilding effort that did take place following the 1972 earthquake created new residential areas east-south-east of the city centre... This gives the city the appearance of a deformed octopus. The tentacles of the octopus reach out along major transport arteries away from the old centre, but the octopus's body is riddled with gaping holes."

8 Ver La Prensa del 10 de mayo de 2004 (artículo disponible en línea por el sitio Internet http://www.laprensa.com.nil archivo/2004/mayo/10/nacionales/nacionales-20040510-07.html, consultado el 23 de junio de 2004).

9 Texto original: "a 'palimpsest' of past forms superimposed upon each other, and a 'collage' of current uses".

10 Texto original: "determined efforts by the 'Miami boys' (as they are called) ...to recreate their cherished Miami social and cultural 'scene'."

11 Nicaragua está brutalmente polarizada entre ricos y pobres. Tiene el quinto coeficiente más desigual de Gini del mundo, según el Banco Mundial (2001: 70-72, tabla 2.8), una situación que empeora porque el país es sumamente pobre, "de modo que, a la desgracia de que el pastel está mal repartido, se añade la desagradable sorpresa de que no hay pastel, sino un diminuto panecillo" (Rocha, 2002). La minoría rica incluye grandes familias tradicionalmente notables, pero también una mezcla de nuevos profesionales, hombres de negocios, intelligentsia, exiliados que han regresado y extranjeros que trabajan principalmente para las ONGs y las agencias internacionales de desarrollo. Es difícil estimar el peso numérico de estos diferentes grupos, pero ninguno es muy grande y, por lo tanto, tiene más sentido considerarlos genéricamente como "elites urbanas".

12 Texto original: "given the anecdotal information on violence as portrayed in the Nicaraguan press and the general perception of violence in Nicaraguan society, these figures are suspiciously low".

13 Ver el informe del Nicaragua Network News Service, vol. 9, no. 6, 5-11 de febrero de 2001, disponible en línea por el sitio Internet http://www.tulane.edu/ libweb/RESTRICTED/NICANEWS/2001/2001 0205.txt, consultado el 16 de junio de 2004.

14 No se sugiere que los pobres no trabajan en estas oficinas o que nunca usen el aeropuerto. Sin embargo, estos nuevos rasgos no fueron desarrollados para pobres. Por ejemplo, la remodelación del aeropuerto de Managua incluyó la construcción de una lujosa tienda libre de impuestos, abastecida casi exclusivamente con artículos importados que, obviamente, no están al alcance de viajeros empobrecidos. Además, la mayoría de los nuevos mostradores que fueron supuestamente construidos 
para minimizar el tiempo de espera de los pasajeros parecen casi exclusivamente servir a los pasajeros de primera clase 0 clase de negocios.

15 Estas estadísticas provienen de La Prensa del 11 de marzo de 2003 (artículo disponible en línea por el sitio Internet http:// www.laprensa.com.ni/cronologico/2003/marzo/11/cartas/cartas-20030311-05.html, consultado el 14 de julio de 2004), Cajina (2000: 169), La Prensa del 3 de marzo de 2003 (artículo disponible en línea por el sitio Internet http://www.laprensa.com.ni/ cronologico/2003/marzo/03/nacionales/nacionales-20030303018.html, consultado el 18 de julio de 2004), La Prensa del 14 de agosto de 2000 (artículo disponible en línea por el sitio Internet http://www.laprensa.com.ni/cronologico/2000/agosto/14/ nacionales/nacionales-20000814-05.html, consultado el 20 de julio de 2004) y datos en línea de la Policía Nacional de Nicaragua disponibles por el sitio Internet http://ww.policia.gob.ni/estadisticas/dosier2003/dosier9.htm, consultado el 18 de julio de 2004.

16 Texto original: "the wealthy ...shield themselves as much as possible from crime and other social problems, constructing higher walls and better security systems for their homes and hiring armed guards to patrol their neighborhoods. In doing so, they create segregated enclaves that, in Managua as elsewhere in Latin America, alter the character of public space and public life and enforce rules of inclusion and exclusion..., [with] the streets of Managua ... left to those who cannot afford to retreat to enclaves".

17 Texto original: "instead of envisioning roads as neutral lines ...going from ... point a to point b, they should be visualized as stretched-out places where intersecting social relations cluster and adhere".

18 Ver El Nuevo Diario del 29 de enero de 2000 (artículo disponible en línea por el sitio Internet http://www-ni.elnuevodiario. com.ni/archivo/2000/enero/29-enero-2000/opinion/opinion6.html, consultado el 23 de junio de 2004).

19 Ver La Prensa del 11 de octubre de 2001 (artículo disponible en línea por el sitio Internet http://www-ni.laprensa.com. ni/archivo/2001/octubre/11/nacionales/nacionales-20011011-07.html. consultado el 18 de mayo de 2004).

20 Ver La Prensa del 31 de mayo 2004 (artículo disponible en línea por el sitio Internet http://www.laprensa.com.ni/archivo/2004/ mayo/31/nacionales/nacionales-20040531-02.html, consultado el 1 de junio de 2004).

21 Ver también los datos en línea del Instituto Nicaragüense de Estadísticas y Censos (INEC) por el sitio Internet http://www. inec.gob.ni/estadisticas/sociodemografico/parqueautomotor.pdf (consultado el 10 de julio de 2004).

22 Ver La Prensa del 29 de enero del 2004 (artículo disponible en línea por el sitio Internet http://www-ni.laprensa.com.ni/ cronologico/2004/enero/29/sucesos/sucesos-20040129-04.html, consultado el 18 de mayo de 2004).

23 Ver La Prensa del 19 de octubre de 2000 (artículo disponible en línea por el sitio Internet http://www-ni.laprensa.com. ni/cronologico/2000/octubre/19/sucesos/sucesos-20001019-01.html, consultado el 18 de mayo de 2004) y La Prensa del 16 de abril de 2004 (artículo disponible en línea por el sitio Internet http://www-ni.laprensa.com.ni/cronologico/2004/abril/16/ sucesos/sucesos-20040416-01.html, consultado el 18 de mayo de 2004).

24 Texto original: "all cities attempt to govern their constituent spaces and those who live there, although to variable extents".

25 Texto original: "the extent to which an actor is 'anchored' in particular territories or places".

26 Texto original: "how social groups relate to each other in the space of the city".

27 Texto original: "worlds of inequality, alienation and injustice".

\section{Referencias bibliográficas}

- BABB, F. E. (2001). After Revolution: Mapping Gender and Cultural Politics in Neoliberal Nicaragua, University of Texas Press, Austin.

- BEALL, J. (2002). La gente detrás de los muros: inseguridad, identidad y comunidades amuralladas en Johannesburgo. Documento de Trabajo de Programa de Estados en Crisis, núm. 10, Programa de Estados en Crisis, Londres. Disponible en World Wide Web: http:// www.crisisstates.com/download/wp/Spanish/WP10.pdf.

- CAJINA, R. J. (2000). "Nicaragua: de la seguridad del Estado a la inseguridad ciudadana", en A. Serbin y D. Ferreyra (comp.), Gobernabilidad Democrática y Seguridad Ciudadana en Centroamérica: El caso de Nicaragua, CRIES, Managua.

- CALDEIRA, T. P. R. (1996). "Building up Walls: The New Pattern of Spatial Segregation in São Paulo”, International Social Science Journal, núm. 147, pág. 55-66, Paris.

- CALDEIRA, T. P. R. (1999). "Fortified enclaves: The new urban segregation”, en J. Holston (comp.), Cities and Citizenship, Duke University Press, Durham. 
- CALDEIRA, T. P. R. (2000). City of Walls: Crime, Segregation and Citizenship in Sao Paulo, University of California Press, Berkeley

- CALL, C. (2000). Sustainable Development in Central America: The Challenges of Violence, Injustice and Insecurity, Documento de Trabajo "CA 2020”, núm. 8, Institut für Iberoamerika-Kunde, Hamburgo

- CHAMORRO, C. F. (2002). "Edificio Pellas fue exonerado del 15\% IGV”. Confidencial núm. 288, 5-11 de mayo, Managua (citado el 26 de julio de 2004). Disponible en World Wide Web: http://www.confidencial.com.ni/2002-288/deportada1-288.html.

- DÍAZ LACAYO, A. (1998). "Nicaragua briefs". Envío in English núm. 204, julio, Managua, (citado el 25 de julio de 2004). Disponible en World Wide Web: http://www.envio.org.ni/ articulo.php?id $=1344$.

- DREWE, P. (1986). "Integrated Upgrading of Marginal Areas in Managua”. Cities, vol. 3, núm. 4, Amsterdam, pág. 333-349.

- GIDDENS, A. (1990). The Consequences of Modernity, Polity Press, Cambridge.

- GODNICK, W.; Muggah, R. y Waszink, C. (2002). Stray Bullets: The Impact of Small Arms Misuse in Central America, Documento de Trabajo, serié "occasional", núm. 5, Small Arms Survey, Ginebra. Disponible en World Wide Web: http://www.smallarmssurvey.org/ publications/occasional.htm.

- GRANERA, A. y CUAREZMA T, (1997). Evolución del delito en Nicaragua (1980-1995), Editorial UCA, Managua.

-GRIGSBY, W. (2003). "La policía en su laberinto: trampas, claves y pistas”. Envío, núm. 257, agosto, Managua (citado el 19 de julio de 2004). Disponible en World Wide Web: http://www. envio.org.ni/articulo.php?id=1271.

- HARVEY, D. (1973). Social Justice and the City, Edward Arnold, Londres.

- HARVEY, D. (1990). The Condition of Postmodernity: An Enquiry into the Origins of Cultural Change. Blackwell, Oxford.

- HARVEY, D. (2003). “The Right to the City”, International Journal of Urban and Regional Research, vol. 27, núm. 4, pág. 939-41, Oxford.

-HESS, M. (2004). "Spatial relationships? Towards a Reconceptualization of Embeddedness". Progress in Human Geography, vol. 28, núm. 2, Londres, pág. 165-186.

- INSTITUTO NICARAGÜENSE DE FOMENTO MUNICIPAL (INIFOM). (2003). Ficha Municipal: Managua, documento consultado en línea el 21 de julio del 2004 por el World Wide Web: http://www.inifom.gob.ni/ArchivosPDF/Managua2.pdf.

- JACKSON, K. T. (1985). Crabgrass Frontier: The Suburbanization of the United States, Oxford University Press, Nueva York.

- LASCH, C. (1995). The Revolt of the Elites and the Betrayal of Democracy, W. W. Norton, Nueva York.

- LEONARDI, R. (2001). Nicaragua Handbook, Footprint Handbooks, Bath.

- LOW, S. (2001). "The Edge and the Center: Gated Communities and the Discourse of Fear", American Anthropologist, vol. 103, núm. 1, Nueva York, pág. 45-58.

- MANGIN, W. (1970). Peasants in Cities, Houghton Mifflin, Boston.

- MERRY, S. E. (2001). "Spatial Governmentality and the New Urban Social Order: Controlling Gender Violence through Law”. American Anthropologist, vol. 103, núm. 1, Nueva York, pág. 16-29.

- MINISTERIO DE LA VIVIENDA Y ASENTAMIENTOS HUMANOS (MINVAH), (1980).

Programa Integral de 2,800 Viviendas para Managua, Managua. 
- ORGANIZACIÓN PANAMERICANA POR LA SALUD, (1998). Health in the Americas, vol. 2, Washington.

- PARK, R. E. et al. (1925). The City: Suggestions for Investigation of Human Behavior in the Urban Environment, University of Chicago Press, Chicago.

- PROGRAMA DE LAS NACIONES UNIDAS PARA EL DESARROLLO (PNUD), (2002). El Desarrollo Humano en Nicaragua: las condiciones de la esperanza, PNUD, Managua.

- RODGERS, D. (2006). "Living in the Shadow of Death: Violence, Pandillas and Social Disintegration in Contemporary Urban Nicaragua, 1996-2002", Journal of Latin American Studies, vol. 38, núm. 2, febrero (en prensa).

- SERBIN, A. y D. FERREYRA (eds), (2000). Gobernabilidad Democrática y Seguridad Ciudadana en Centroamérica: el caso de Nicaragua, CRIES, Managua.

- SMART, A. (2001). "Unruly Places: Urban Governance and the Persistence of Illegality in Hong Kong's Urban Squatter Areas”. American Anthropologist, vol. 103, núm. 1, Nueva York, pág. 30-44.

- SVAMPA, M. (2001). Los que ganaron: La vida en los countries y barrios privados. Editorial Biblos, Buenos Aires

- VANCE, I. (1985). "More than bricks and mortar: women's participation in self-help housing in Managua, Nicaragua", en C. Moser y L. Peake (comp.), Women, Human Settlements and Housing, Tavistock, Londres.

- WALL, D. L. (1996). “City Profile: Managua”, Cities, vol. 13, núm. 1, Amsterdam, pág. 4552.

- WHISNANT, D. E. (1995). Rascally Signs in Sacred Places: The Politics of Culture in Nicaragua, Chapel Hill: University of North Carolina Press.

- WILSON, F. (2004). "Towards a Political Economy of Roads: Experiences from Peru”, Development and Change, vol. 35, núm. 3, La Haya, pag. 525-46. 